15th International Spin Physics Symposium

(SPIN 2002)

Upton, New York

September 9-14, 2002

\title{
The Relativistic Stern-Gerlach Interaction as a Tool for Attaining the Spin Separation*
}

\author{
P. Cameron ${ }^{*}$, M. Conte ${ }^{\dagger}$, A. U. Luccio*, W. W. MacKay*, M. Palazzi ${ }^{\dagger}$ and \\ M. Pusterla** \\ * Brookhaven National Laboratory, Upton, NY 11973, USA. \\ $\dagger$ 'Dipartimento di Fisica dell'Università di Genova, INFN Sezione di Genova, Via Dodecaneso 33, \\ 16146 Genova, Italy. \\ ** Dipartimento di Fisica dell'Università di Padova, INFN Sezione di Padova, Via Marzolo 8, \\ 35131 Padova, Italy.
}

\begin{abstract}
The relativistic Stern-Gerlach interaction is here considered as a tool for obtaining the spin state separation of an unpolarized (anti)proton beam circulating in a ring. Drawbacks, such as spin precessions within the TE rf cavity, spurious kicks due to the transverse electric field and, worst of all, filamentation in the longitudinal phase plane are analyzed. Possible remedies are proposed and their feasibility is discussed.
\end{abstract}

\section{INTRODUCTION}

We have exhaustively demonstrated [1] that the relativistic Stern-Gerlach interaction can play a decisive role in accomplishing the spin states separation of a high energy unpolarized beam of protons and, possibly, of antiprotons, since the single cavity crossing energy kick is

$$
d U \simeq 2 \gamma^{2} B_{0} \mu^{*}
$$

where $\gamma$ is the Lorentz factor, $B_{0}$ is the peak magnetic field in the cavity and $\mu^{*}=$ $\left|\vec{\mu}^{*}\right|$ is the particle magnetic moment: $1.41 \times 10^{-26} \mathrm{JT}^{-1}$ for (anti)protons and $9.28 \times$ $10^{-24} \mathrm{JT}^{-1}$ for electrons and positrons.

After having crossed $N_{\text {cav }}$ cavities and completed $N_{\text {turns }}$ revolutions, particles with opposite spin states should be gathered in couples of bunches exhibiting an energy separation

$$
\Delta U \simeq 4 N_{\text {turns }} N_{\text {cav }} \gamma^{2} B_{0} \mu^{*}
$$

and a momentum spread

$$
\frac{\Delta p}{p}=\frac{1}{\beta^{2}} \frac{\Delta U}{U} \simeq 4 N_{\text {turns }} N_{\text {cav }} \frac{B_{0}}{B_{\infty}} \gamma
$$

with $B_{\infty}=\frac{m c^{2}}{\mu^{*}} \simeq 10^{16} T$ for (anti)protons. The number of revolutions and the time interval required for reaching the value of the design momentum spread are

*Work performed under the auspices of US Department of Energy CP675, Spin 2002: 15th Int'l. Spin Physics Symposium and Workshop on Polarized Electron

Sources and Polarimeters, edited by Y. I. Makdisi, A. U. Luccio, and W. W. MacKay (C) 2003 American Institute of Physics 0-7354-0136-5/03/\$20.00 
TABLE 1. RHIC, HERA and LHC Parameters

\begin{tabular}{cccc}
\hline & RHIC & HERA & LHC \\
\hline $\mathrm{E}(\mathrm{GeV})$ & 250 & 820 & 7000 \\
$\gamma$ & 266.5 & 874.2 & 7462.7 \\
$\tau_{\mathrm{rev}}(\mu s)$ & 12.8 & 21.1 & 88.9 \\
$\Delta \mathrm{p} / \mathrm{p}$ & $4.1 \times 10^{-3}$ & $5 \times 10^{-5}$ & $1.05 \times 10^{-4}$ \\
$N_{\mathrm{SS}}$ & $6.67 \times 10^{9}$ & $2.48 \times 10^{7}$ & $1.76 \times 10^{6}$ \\
$\Delta \mathrm{t}$ & $23.7 \mathrm{hr}$ & $523 \mathrm{~s}$ & $156 \mathrm{~s}$ \\
\hline \multicolumn{4}{|}{} \\
$N_{\mathrm{SS}}=\frac{B_{\infty}}{4 N_{\mathrm{cav}} B_{0} \gamma}\left(\frac{\Delta p}{p}\right)_{\text {ring }}$, & and $\Delta t=N_{\mathrm{SS}} \tau_{\mathrm{rev}}$.
\end{tabular}

By applying Eq. (4) to three rings, either operating or under development, we find the data gathered in Table 1. From the last row, we can ascertain how the LHC [2] splitting time is rather short making us quite optimistic. Nevertheless, it is wise to analyze all the drawbacks which can haunt the proposed procedure.

\section{SPURIOUS EFFECTS}

An effect to be considered is the one regarding the influence on the spin precession of the if fields.

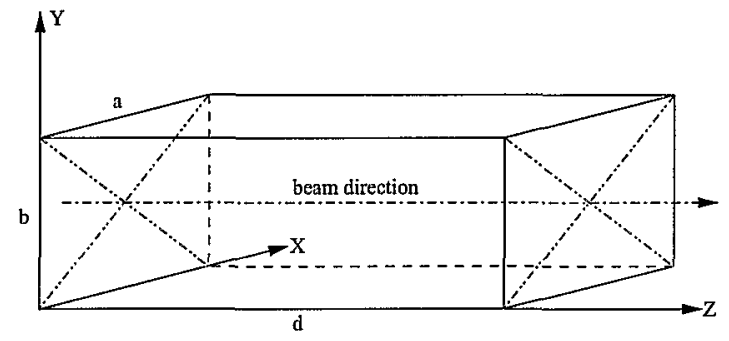

FIGURE 1. Rectangular cavity.

We recall the Thomas-BMT equation

$$
\frac{d \vec{S}}{d t}=\vec{\Omega}_{s} \times \vec{S}, \quad \text { or } \quad \frac{d \vec{P}}{d t}=\vec{\Omega}_{s} \times \vec{P}
$$

with

$$
\vec{\Omega}_{s}=-\frac{e}{\gamma m}\left\{(1+a \gamma) \vec{B}-(\gamma-1) a \frac{(\vec{v} \cdot \vec{B})}{v^{2}} \vec{v}+\gamma\left[\left(a+\frac{1}{\gamma+1}\right) \frac{\vec{E} \times \vec{v}}{c^{2}}\right]\right\},
$$

where $\vec{P}=\left(P_{x}, P_{y}, P_{z}\right)$ is the beam polarization built up by the Stern-Gerlach energy kicks. The proposed $3 \mathrm{GHz} \mathrm{TE}_{011}$ mode, inside a rectangular cavity (see Fig. 1), is characterized by the fields 


$$
\begin{aligned}
& \vec{B}=\left(\begin{array}{c}
0 \\
-B_{0} \frac{b}{d} \sin \left(\frac{\pi y}{b}\right) \cos \left(\frac{\pi z}{d}\right) \cos (\omega t) \\
B_{0} \cos \left(\frac{\pi y}{b}\right) \sin \left(\frac{\pi z}{d}\right) \cos (\omega t)
\end{array}\right), \\
& \vec{E}=\left(\begin{array}{c}
-\omega B_{0} \frac{b}{\pi} \sin \left(\frac{\pi y}{b}\right) \sin \left(\frac{\pi z}{d}\right) \sin (\omega t) \\
0 \\
0
\end{array}\right) .
\end{aligned}
$$

The effects of these fields, which are expected to be negligible, will be analyzed by means of computer simulation, starting with a polarization $\vec{P}_{0}=\left(0, P_{0 y}, 0\right)$ at the cavity entrance and looking for the the polarization state at the cavity exit.

Another effect to be considered is the interaction between the cavity's electric field and the particle's electric charge. We have already [1] demonstrated that, after a single cavity crossing, the energy exchange is

$$
\Delta U_{E}=\left[e \omega B_{0} \frac{b d}{\pi^{2}} \frac{\beta^{2}}{\beta_{\mathrm{ph}}^{2}-\beta^{2}} \sin \left(\frac{\beta_{\mathrm{ph}}}{\beta} \pi\right)\right] x^{\prime},
$$

where $\beta_{\mathrm{ph}}=\sqrt{1+(d / b)^{2}}$ is a function of the cavity dimensions $b$ and $d$ (see Fig. 1). For ultrarelativistic particles $\beta_{\mathrm{ph}}$ equal to an integer, Eq. (9) reduces to

$$
\Delta U_{E}= \pm\left[e \omega B_{0} \frac{b d}{2 \pi} \frac{\beta_{\mathrm{ph}}}{\beta_{\mathrm{ph}}^{2}-\beta^{2}}\left(\frac{\varepsilon}{\beta_{\mathrm{ph}}}+\frac{1}{\gamma^{2}}\right)\right] x^{\prime},
$$

having accounted for the error $\varepsilon$ in $\beta_{\mathrm{ph}}$. The quantity within square brackets is very small; besides the trajectory slope $x^{\prime}$ averages continuously to zero every few turns, due to required incoherence of betatron oscillations. However, a rather pleonastic computer simulation confirms the insubstantiality of such an effect. In fact, by defining

$$
\delta x=(x)_{\mathrm{rf}}-(x)_{\text {norf }},
$$

where $(x)_{\mathrm{rf}}$ is the path run by the particle after having crossed the cavity with the radio frequency on, and $(x)_{\text {norf }}$ is the same path with the rf switched off, we may assess the displacement through the cavity for the four initial conditions at the entrance: $\left( \pm x_{0}, 0\right)$ and $\left(0, \pm x_{0}^{\prime}\right)$, where the quantities $x_{0}$ and $x_{0}^{\prime}$ are compatible with the LHC normalized emittance $\varepsilon^{*}=3.75 \mu \mathrm{m}$. The plot in Fig. 2a (the same for all four cases) exhibits a displacement of $5 \mathrm{~nm}$, i.e. an actually negligible effect.

\section{FILAMENTATION IN THE LONGITUDINAL PHASE PLANE}

We have already discussed [3] how the plots in the synchrotron oscillations phase plane are distorted due to the typical non linearity of the phase oscillations equation

$$
\frac{d^{2} \phi}{d t^{2}}+\Omega_{s}^{2} \sin \phi=0 \quad \text { (stationary bucket case), }
$$



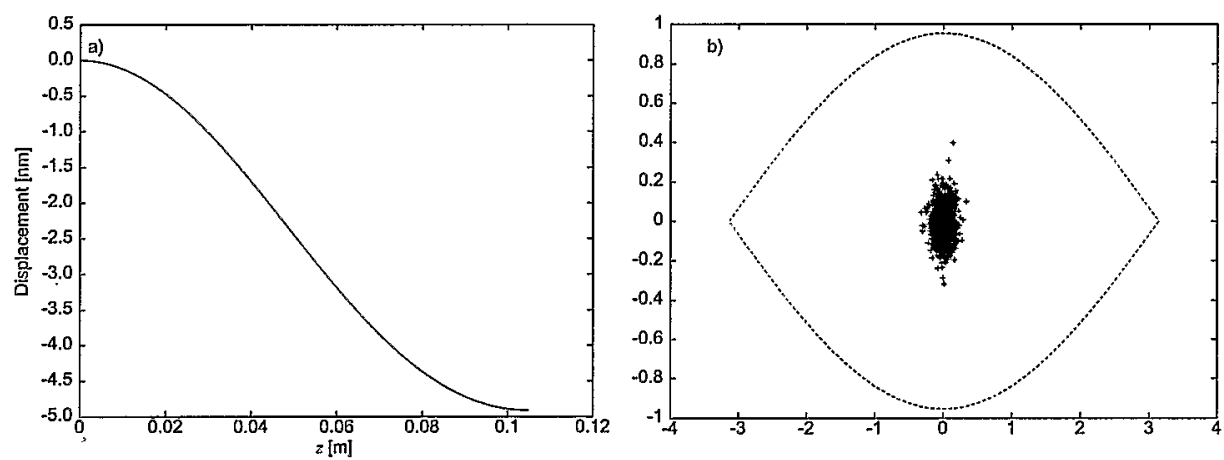

FIGURE 2. a) Particle trajectory inside the cavity. b) Longitudinal phase space of initial bunch.

TABLE 2. LHC Parameters at Collision

\begin{tabular}{lcc}
\hline & Values & Unit \\
\hline Revolution frequency & 11.2455 & $\mathrm{kHz}$ \\
rf frequency & 400.7 & $\mathrm{MHz}$ \\
Harmonic number $h$ & 35640 & \\
If voltage $V_{\text {If }}$ & 16 & $\mathrm{MV}$ \\
Synchrotron period $\tau_{s}$ & 0.042 & $\mathrm{~s}$ \\
Transition parameter $\eta_{\mathrm{tr}}$ & $3.47 \times 10^{-4}$ & \\
Bunch duration & 0.28 & $\mathrm{~ns}$ \\
Bunch length & 8.39 & $\mathrm{~cm}$ \\
\hline
\end{tabular}

with

$$
\Omega_{s}=\omega_{s} \sqrt{\frac{h\left|\eta_{\mathrm{tr}}\right|}{2 \pi \beta^{2} \gamma} \frac{q V_{\mathrm{rf}}}{m c^{2}}} \simeq \omega_{s} \sqrt{\frac{h \mid \eta_{\mathrm{tr}}}{2 \pi \gamma} \frac{q V_{\mathrm{rf}}}{m c^{2}}} \quad \text { (ultrarelativistic) }
$$

where

$$
\eta_{\mathrm{tr}}=\gamma^{-2}-\alpha_{p}=\gamma^{-2}-\gamma_{\mathrm{tr}}^{-2}
$$

is the phase-slip factor ( $\alpha_{p}=$ momentum compaction factor), $h$ is the harmonic number and $V_{\text {rf }}$ is the peak if voltage. The synchrotron period is

$$
\tau_{s}=\frac{2 \pi}{\Omega_{s}}=\tau_{\mathrm{rev}} \sqrt{\frac{2 \pi \beta^{2} \gamma}{h \mid \eta_{\mathrm{tr}}} \frac{V_{p}}{V_{\mathrm{rf}}}} \simeq \tau_{\mathrm{rev}} \sqrt{\frac{2 \pi \gamma}{h \mid \eta_{\mathrm{tr}}} \frac{V_{p}}{V_{\mathrm{rf}}}} \quad \text { (ultrarelativistic) }
$$

with $V_{p}=938 \mathrm{MV}$ for (anti)protons.

Concentrating our attention on LHC, we gather in Table $2 \mathrm{a}$ few parameters of interest which will be used together with the data in the third column of Table 1. Starting with a bunch like the one illustrated in Fig. 2b, the simulation program shows (see Fig. 3a) that the filamentation begins scarcely after 10 synchrotron periods, i.e. after about $0.42 \mathrm{~s}$ : a time much smaller than $(\Delta t)_{\mathrm{LHC}}=156 \mathrm{~s}$ shown in Table 1 . However, the cavity's magnetic field $B_{0}=0.1 \mathrm{~T}$ could be increased by a factor of 10 , since the associated electric field would be $300 \mathrm{MV} / \mathrm{m}$, a value already realized in TM cavities. Besides, it would not be impossible to lower the momentum dispersion down to $10^{-5}$, perhaps at expense of beam intensity. With these values, a new $(\Delta t)_{\mathrm{LHC}}=1.56 \mathrm{~s}$ will result. After 

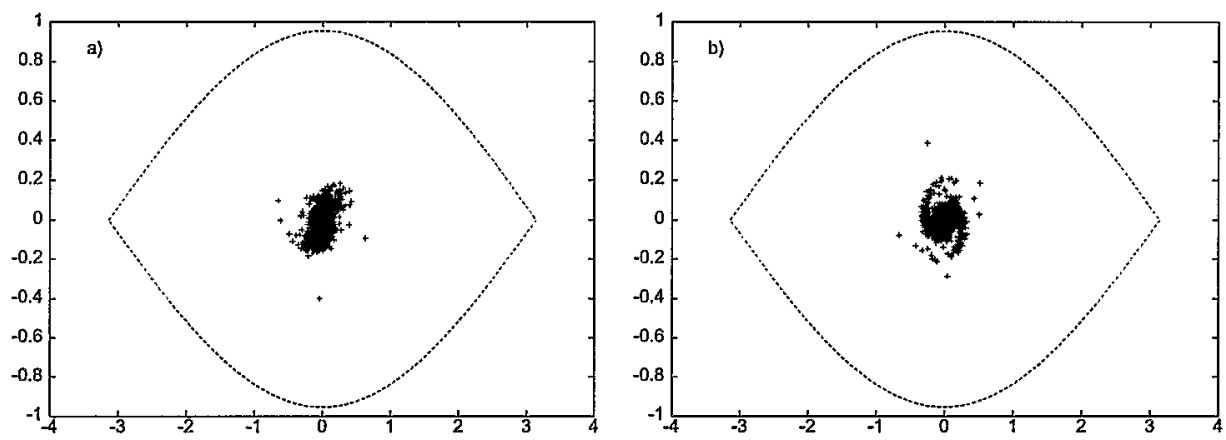

FIGURE 3. a) Filamentation after 10 synchrotron periods. b) Filamentation after 40 synchrotron periods with the more stringent requirements discussed in the text.

40 synchrotron periods, i.e. after $1.68 s$, the filamentation is not too bad, as illustrated in Figs. 3b. This means that the desired spin-state separation could occur, although with an efficiency less than $100 \%$ due to the "tails" generated by the filamentation phenomenon. Notwithstanding, it is worthwhile to note that there are not so many particles in the tails.

\section{CONCLUSIONS}

We have demonstrated that the self polarization of the LHC high energy protons might be attained by making use of the time varying Stern-Gerlach interaction. The bunch length of $8.39 \mathrm{~cm}$ (See Table 2.) fits very well the TE wavelength $\lambda=10 \mathrm{~cm}$. This of course assumes that the LHC lattice would be capable of maintaining the polarization of a stored beam; however without the addition of several snakes, this is perhaps illusory. As should be clear, what found here is specific of this particular machine. For other rings, e.g. such as Tevatron [4], things have to be reconsidered, perhaps exploiting other physical properties of particle accelerators.

Since most high energy colliders are not designed with polarization in mind, it becomes problematic to refit them later for polarized beams. Perhaps the right approach would be to design a conceptual collider optimized for polarized beams; then the ascertained concepts could be implemented up front in designs for future machines.

\section{REFERENCES}

1. M. Conte et al., INFN/TC-00/03 (2000), (http:xxx.lanl.gov/listphysics/0003, preprint 0003069); M. Conte et al., ICFA Beam Dynamics Newsletter, 24, 66 (2001), (http://wwwslap.cern.ch/icfa/);P. Cameron et al., Proc. of the SPIN2000 Symposium, AIP Conf. Proc. 570, 785 (2001).

2. See, for instance, http://lhc-new-homepage.web.cem.ch/lhc-new-homepage/

3. M. Conte, W.W. MacKay and R. Parodi, BNL-52541, (1997).

4. http://www-bdnew.final.gov/tevatron/ 